J. DIFFERENTIAL GEOMETRY

93 (2013) 379-400

\title{
ON A GENERALIZATION OF A THEOREM OF MCDUFF
}

\author{
G. Deltour
}

\begin{abstract}
We study the symplectic structure of the holomorphic coadjoint orbits, generalizing a theorem of McDuff on the symplectic structure of Hermitian symmetric spaces of noncompact type.
\end{abstract}

\section{Introduction}

In this article, we are interested in the symplectic structure of holomorphic coadjoint orbits.

Let $\mathrm{G}$ be a noncompact, connected, real semisimple Lie group with finite center, and let $K$ be a maximal compact subgroup of $G$. We denote by $\mathfrak{g}$ and $\mathfrak{k}$ the Lie algebras of $G$ and $K$ respectively. The maximal compact subgroup $K$ is connected and corresponds to a Cartan decomposition $\mathfrak{g}=\mathfrak{k} \oplus \mathfrak{p}$ on the Lie algebra level; see for instance [6]. Assume that $G / K$ is a Hermitian symmetric space, that is, there exists a $G$ invariant complex structure on the manifold $G / K$. Let $T$ be a maximal torus in $K$ and $\mathfrak{t}$ its Lie algebra. We fix a Weyl chamber $\mathfrak{t}_{+}^{*} \subset \mathfrak{t}^{*}$ for the compact group $K$. We recall that a coadjoint orbit $\mathcal{O}$ of the noncompact group $G$ is called elliptic if $\mathcal{O}$ intersects $\mathfrak{k}^{*}$.

Any coadjoint orbit $\mathcal{O}$ carries a canonical $G$-invariant symplectic form $\Omega_{\mathcal{O}}$, called the Kirillov-Kostant-Souriau symplectic form on $\mathcal{O}$. If $\mathcal{O}=$ $G \cdot \lambda$ is the coadjoint orbit through $\lambda \in \mathfrak{g}^{*}$, the symplectic form $\Omega_{G \cdot \lambda}$ is defined above $\lambda$ by

$$
\left.\left(\Omega_{G \cdot \lambda}\right)\right|_{\lambda}(X, Y):=\langle\lambda,[X, Y]\rangle, \text { for all } X, Y \in \mathfrak{g} / \mathfrak{g}_{\lambda},
$$

where we identify canonically the tangent space $T_{\lambda}(G \cdot \lambda)$ with the vector space $\mathfrak{g} / \mathfrak{g}_{\lambda}$. For instance, considering the homogeneous space $G / K$ as the coadjoint orbit of some generic element of the center of $\mathfrak{k}^{*}$, the Kirillov-Kostant-Souriau form defines a natural $G$-invariant symplectic structure on the Hermitian symmetric space $G / K$, which is compatible with the $G$-invariant complex structure on $G / K$.

Actually, the Hermitian symmetric spaces form a part of a much larger family of Kähler coadjoint orbits, called holomorphic coadjoint

Received 2/24/2011. 
orbits. They are the coadjoint orbits which are related to the holomorphic discrete series of $G$.

More precisely, a holomorphic coadjoint orbit is an elliptic coadjoint orbit $\mathcal{O}$ of $G$ which carries a canonical $G$-invariant Kähler structure, compatible with the Kirillov-Kostant-Souriau symplectic structure on $\mathcal{O}$. The holomorphic coadjoint orbits are parametrized by a subchamber $\mathcal{C}_{\text {hol }}$ of $\mathfrak{t}_{+}^{*}$, called the holomorphic chamber. This chamber can be formally defined using noncompact roots of $G$; see section 2.2. This definition has the following consequence: if $\lambda \in \mathcal{C}_{\text {hol }}$, then the stabilizer $G_{\lambda}$ of $\lambda$ in $G$ is compact (that is, the coadjoint orbit $G \cdot \lambda$ is strongly elliptic). In particular, when $G_{\lambda}=K$, the holomorphic coadjoint orbit $G \cdot \lambda$ coincides with the Hermitian symmetric space $G / K$.

In the $80 \mathrm{~s}, \mathrm{McDuff}[8]$ proved that any Hermitian symmetric space of noncompact type is diffeomorphic, as a symplectic manifold, to a symplectic vector space. This yields a global version of Darboux's theorem for every Hermitian symmetric space.

Our purpose is to extend McDuff's Theorem to any holomorphic coadjoint orbit.

First of all, we introduce our symplectic model for general holomorphic coadjoint orbits of $G$, which extends the symplectic vector space model obtained for the Hermitian symmetric space case.

Let $\lambda \in \mathcal{C}_{\text {hol }}$. Using the Cartan decomposition on the Lie group $G$, we know that the manifold structure of $G \cdot \lambda$ is $K$-equivariantly diffeomorphic to the product $K \cdot \lambda \times \mathfrak{p}$, on which $K$ acts diagonally. These two manifolds admit canonical symplectic structures $\Omega_{G \cdot \lambda}$ and $\Omega_{K \cdot \lambda \times \mathfrak{p}}=\Omega_{K \cdot \lambda} \oplus \Omega_{\mathfrak{p}}$ (direct product of two symplectic forms), where $\Omega_{K \cdot \lambda}$ denotes the Kirillov-Kostant-Souriau symplectic form on the compact coadjoint orbit $K \cdot \lambda$, and $\Omega_{\mathfrak{p}}$ is a $K$-invariant constant symplectic form on the vector space $\mathfrak{p}$ (its definition is given in section 2.3).

The main goal of this article is to prove the following generalization of McDuff's Theorem, conjectured by Paradan in [11].

Theorem 1.1. Let $\lambda \in \mathcal{C}_{\text {hol }}$. Then there exists a $K$-equivariant diffeomorphism from $G \cdot \lambda$ onto $K \cdot \lambda \times \mathfrak{p}$ which takes the symplectic form $\Omega_{G \cdot \lambda}$ on $G \cdot \lambda$ to the symplectic form $\Omega_{K \cdot \lambda \times \mathfrak{p}}$ on $K \cdot \lambda \times \mathfrak{p}$.

REMARK 1.1. As we will see in the proof of Theorem 1.1, we can assume that the diffeomorphism, obtained in the above statement, also satisfies that each element $k \lambda \in K \cdot \lambda \subset G \cdot \lambda$ is sent to $(k \lambda, 0) \in K \cdot \lambda \times \mathfrak{p}$.

The symplectic manifolds $\left(G \cdot \lambda, \Omega_{G \cdot \lambda}\right)$ and $\left(K \cdot \lambda \times \mathfrak{p}, \Omega_{K \cdot \lambda \times \mathfrak{p}}\right)$ actually have Hamiltonian $K$-manifold structures given respectively by the moment maps $\Phi_{G \cdot \lambda}:\left.\xi \in G \cdot \lambda \subseteq \mathfrak{g}^{*} \mapsto \xi\right|_{\mathfrak{k}} \in \mathfrak{k}^{*}$ (that is, the standard orbit projection of $G \cdot \lambda$ on $\mathfrak{k}^{*}$ ), and

$$
\begin{aligned}
\Phi_{K \cdot \lambda \times \mathfrak{p}}: \quad K \cdot \lambda \times \mathfrak{p} & \rightarrow \mathfrak{k}^{*} \\
(\xi, v) & \mapsto\left(X \in \mathfrak{k} \mapsto\langle\xi, X\rangle+\frac{1}{2} \Omega_{\mathfrak{p}}(v,[X, v])\right) .
\end{aligned}
$$


Let $\Delta_{K}(G \cdot \lambda):=\Phi_{G \cdot \lambda}(G \cdot \Lambda) \cap \mathfrak{t}_{+}^{*}$ and $\Delta_{K}(K \cdot \lambda \times \mathfrak{p}):=\Phi_{K \cdot \lambda \times \mathfrak{p}}(K \cdot \lambda \times$ $\mathfrak{p}) \cap \mathfrak{t}_{+}^{*}$ denote the associated moment polyhedra. Theorem 1.1 has the following direct consequence, originally proved by Nasrin (for $\lambda$ in the center of $\left.\mathfrak{k}^{*}\right)$ and Paradan in totally different ways $[\mathbf{1 0}, \mathbf{1 1}]$.

Corollary 1.2 (Nasrin, Paradan). Let $\lambda \in \mathcal{C}_{\text {hol }}$. Then

$$
\Delta_{K}(G \cdot \lambda)=\Delta_{K}(K \cdot \lambda \times \mathfrak{p}) .
$$

This new description of the moment polyhedron $\Delta_{K}(G \cdot \lambda)$ allows us to describe its faces, using GIT methods on the second setting [2].

This article is completely dedicated to the proof of Theorem 1.1. In section 2, we introduce the notion of holomorphic coadjoint orbit and some other preliminary facts about Cartan decomposition of $G$. The main tool, constructing symplectomorphisms by a Moser argument on a special noncompact setting, is given in section 3 . It uses the properness of the involved moment maps so as to be able to integrate a particular vector field on the noncompact manifold $K \cdot \lambda \times \mathfrak{p}$. Theorem 1.1 is then proved in section 4 for the case of Hermitian symmetric spaces, and section 5 achieves the general proof.

Acknowledgements. I would like to thank Paul-Émile Paradan for our many discussions and his precious help. I thank the referees for their helpful comments which improved the presentation of this article.

\section{Preliminaries}

In this section, we set some definitions and notations. From now on, let $G$ be a noncompact, connected, real semisimple Lie group with finite center, and $\mathfrak{g}$ its Lie algebra.

2.1. Properties of the Cartan decomposition. Here, we recall some facts about Cartan decomposition of $G$, and establish two lemmas. A good exposition on Cartan decomposition of real semisimple Lie groups can be found in $[\mathbf{6}$, Chapter VI].

Since $\mathfrak{g}$ is semisimple, there exists a Cartan involution $\theta$ on $\mathfrak{g}$. We recall that a Cartan involution on the Lie algebra $\mathfrak{g}$ is an involutive Lie algebra automorphism $\theta$ of $\mathfrak{g}$ such that the symmetric bilinear form $B_{\theta}$ defined by

$$
B_{\theta}(X, Y)=-B_{\mathfrak{g}}(X, \theta(Y)), \quad \text { for all } X, Y \in \mathfrak{g},
$$

is positive definite on $\mathfrak{g}$. Here, $B_{\mathfrak{g}}$ denotes the Killing form on $\mathfrak{g}$.

Let $\mathfrak{k}$ (resp. $\mathfrak{p}$ ) be the eigenspace of $\theta$ with eigenvalue 1 (resp. -1 ). From the definitions, we have the Cartan decomposition $\mathfrak{g}=\mathfrak{k} \oplus \mathfrak{p}$ and the inclusions $[\mathfrak{k}, \mathfrak{k}] \subseteq \mathfrak{k},[\mathfrak{p}, \mathfrak{p}] \subseteq \mathfrak{k}$, and $[\mathfrak{k}, \mathfrak{p}] \subseteq \mathfrak{p}$. Then $\mathfrak{k}$ is a Lie subalgebra of $\mathfrak{g}$.

Let $K$ be the connected Lie subgroup of $G$ with Lie algebra $\mathfrak{k}$. Then, $K$ is a maximal compact subgroup of $G$, and we have a $K$-invariant 
diffeomorphism $K \times \mathfrak{p} \rightarrow G,(k, Z) \mapsto e^{Z} k$, known as the Cartan decomposition on the Lie group $G$.

Fix $\lambda \in \mathfrak{k}^{*}$ such that its stabilizer in $G$ is compact, that is, $G_{\lambda}=K_{\lambda}$. Then the Cartan decomposition induces the $K$-invariant diffeomorphism

$$
\begin{aligned}
\Gamma: K \cdot \lambda \times \mathfrak{p} & \longrightarrow G \cdot \lambda \\
(\xi, Z) & \longmapsto e^{Z} \xi .
\end{aligned}
$$

We can identify the tangent bundle of the homogeneous space $G \cdot \lambda$ (resp. $K \cdot \lambda$ ) with the manifold $G \times_{G_{\lambda}} \mathfrak{g} / \mathfrak{g}_{\lambda}\left(\right.$ resp. $\left.K \times_{K_{\lambda}} \mathfrak{k} / \mathfrak{k}_{\lambda}\right)$ by using the diffeomorphism

$$
\begin{aligned}
& G \times \times_{G_{\lambda}} \mathfrak{g} / \mathfrak{g}_{\lambda} \quad \longrightarrow \quad T(G \cdot \lambda) \\
& {\left.\left[g, X \quad \bmod \mathfrak{g}_{\lambda}\right] \longmapsto \frac{d}{d t}\left(g e^{t X} \lambda\right)\right|_{t=0}}
\end{aligned}
$$

(resp. $\left.K \times_{K_{\lambda}} \mathfrak{k} / \mathfrak{k}_{\lambda} \rightarrow T(K \cdot \lambda),\left.\left[k, X \bmod \mathfrak{k}_{\lambda}\right] \mapsto \frac{d}{d t}\left(k e^{t X} \lambda\right)\right|_{t=0}\right)$. Since $\Gamma$ is defined in terms of the exponential function, its derivative will involve the linear endomorphisms of $\mathfrak{g}$

$$
\Psi_{Z}:=\int_{0}^{1} e^{-s \operatorname{ad}(Z)} d s=\sum_{n=0}^{+\infty} \frac{(-1)^{n} \operatorname{ad}(Z)^{n}}{(n+1) !},
$$

defined for all $Z \in \mathfrak{g}$. For $Z \in \mathfrak{g}$, the derivative of exp at $Z$ is given by the equation

$$
\left.\frac{d}{d t}(\exp (Z+t X))\right|_{t=0}=\left.\frac{d}{d t}\left(e^{Z} \exp \left(t \Psi_{Z}(X)\right)\right)\right|_{t=0}, \quad \forall X \in \mathfrak{g} .
$$

See for example [3, Theorem 1.5.3]. We can now compute the derivative of $\Gamma$ at any point of $K \cdot \lambda \times \mathfrak{p}$.

Lemma 2.1. For all $(k \lambda, Z) \in K \cdot \lambda \times \mathfrak{p}$ and all $(X, A) \in \mathfrak{k} / \mathfrak{k}_{\lambda} \oplus \mathfrak{p}$, we have

$$
d \Gamma(k \lambda, Z)([k, X], A)=\left[e^{Z} k, X+\operatorname{Ad}\left(k^{-1}\right) \Psi_{Z}(A)\right] .
$$

Proof. By linearity of $d \Gamma(k \lambda, Z)$, we only have to compute separately the expressions $\left.\frac{d}{d t}\left(e^{Z} k \exp (t X) \lambda\right)\right|_{t=0}$ and $\left.\frac{d}{d t}(\exp (Z+t A) k \lambda)\right|_{t=0}$. But the first term is equal to $\left[e^{Z} k, X\right]$ by definition. Moreover, using equation (2), we have

$$
\left.\frac{d}{d t}(\exp (Z+t A) k \lambda)\right|_{t=0}=\frac{d}{d t}\left(\left.e^{Z} k \exp \left(t \Psi_{\operatorname{Ad}\left(k^{-1}\right) Z}\left(\operatorname{Ad}\left(k^{-1}\right) A\right) \lambda\right)\right|_{t=0},\right.
$$

which is also equal to $\left[e^{Z} k, \Psi_{\operatorname{Ad}\left(k^{-1}\right) Z}\left(\operatorname{Ad}\left(k^{-1}\right) A\right)\right]$. One can easily check that $\Psi_{\operatorname{Ad}\left(k^{-1}\right) Z}\left(\operatorname{Ad}\left(k^{-1}\right) A\right)=\operatorname{Ad}\left(k^{-1}\right) \Psi_{Z}(A)$ from the definition of $\Psi_{Z}$.

q.e.d.

Now, let $Z$ be in $\mathfrak{p}$. Then $\operatorname{ad}(Z)$ is a symmetric endomorphism relative to the inner product $B_{\theta}$, cf. [6, Lemma 6.27]. Since $\Psi_{Z}$ is defined as the convergent series (1), it is also symmetric for $B_{\theta}$. Its eigenvalues are positive real numbers, so $\Psi_{Z}$ is positive definite. 
Note that, for all positive integers $n$, the endomorphism $\operatorname{ad}(Z)^{2 n}$ maps $\mathfrak{k}($ resp. $\mathfrak{p})$ into $\mathfrak{k}($ resp. $\mathfrak{p})$, and $\operatorname{ad}(Z)^{2 n+1}$ maps $\mathfrak{k}$ (resp. $\left.\mathfrak{p}\right)$ into $\mathfrak{p}$ (resp. $\mathfrak{k})$, because of the inclusions $[\mathfrak{k}, \mathfrak{k}] \subseteq \mathfrak{k},[\mathfrak{p}, \mathfrak{p}] \subseteq \mathfrak{k}$, and $[\mathfrak{k}, \mathfrak{p}] \subseteq \mathfrak{p}$. Then we decompose $\Psi_{Z}$ in two parts, $\Psi_{Z}=\Psi_{Z}^{+}+\Psi_{Z}^{-}$, where

$$
\Psi_{Z}^{+}:=\sum_{n=0}^{+\infty} \frac{\operatorname{ad}(Z)^{2 n}}{(2 n+1) !} \text { and } \Psi_{Z}^{-}:=-\sum_{n=0}^{+\infty} \frac{\operatorname{ad}(Z)^{2 n+1}}{(2 n+2) !} .
$$

These linear endomorphisms of $\mathfrak{g}$ are symmetric for $B_{\theta}$, and $\Psi_{Z}^{+}$is also positive definite. Thus $\Psi_{Z}^{+}$is invertible, but $\Psi_{Z}^{-}$is not, since $\Psi_{Z}^{-}(Z)=0$. We can define the linear map

$$
\chi_{Z}:=\Psi_{Z}^{-} \circ\left(\Psi_{Z}^{+}\right)^{-1}: \mathfrak{g} \rightarrow \mathfrak{g} .
$$

In section 5 , we will use the important property of $\chi_{Z}$ stated in the next lemma.

Lemma 2.2. For all $Z \in \mathfrak{p}$, the linear map $\chi_{Z}: \mathfrak{g} \rightarrow \mathfrak{g}$ is symmetric for $B_{\theta}$, and its eigenvalues are in $]-1,1[$.

Proof. Clearly, since $\Psi_{Z}^{+}$is symmetric, $\left(\Psi_{Z}^{+}\right)^{-1}$ is also symmetric. Moreover, $\operatorname{ad}(Z)$ commutes with $\left(\Psi_{Z}^{+}\right)^{-1}$, because it commutes with $\Psi_{Z}^{+}$. Now, from the definition of $\Psi_{Z}^{-}$and the linearity of $\left(\Psi_{Z}^{+}\right)^{-1}, \Psi_{Z}^{-}$ obviously commutes with $\left(\Psi_{Z}^{+}\right)^{-1}$. This implies that $\chi_{Z}=\Psi_{Z}^{-} \circ\left(\Psi_{Z}^{+}\right)^{-1}$ is symmetric for $B_{\theta}$, and then, if $\nu_{1}, \ldots, \nu_{r}$ are the eigenvalues of $\operatorname{ad}(Z)$, a quick calculation shows that the eigenvalues of $\chi_{Z}$ are the real numbers $\left.\left(e^{\nu_{j}}-1\right) /\left(e^{\nu_{j}}+1\right) \in\right]-1,1[$, for $j=1, \ldots, r$.

q.e.d.

2.2. The holomorphic chamber $\mathcal{C}_{\text {hol }}$. We recall that the symmetric space $G / K$ is Hermitian if it admits a complex-manifold structure such that $G$ acts by holomorphic transformations. If $G$ and $K$ satisfies the previous hypotheses (that is, $G$ is noncompact, connected, real semisimple Lie group with finite center, and $K$ a maximal compact subgroup of $G)$, then the following assertions are equivalent:

1) $G / K$ is Hermitian,

2) there exists $z_{0}$ in the center of $\mathfrak{k}$ such that $\left.\operatorname{ad}\left(z_{0}\right)\right|_{\mathfrak{p}} ^{2}=-\left.\mathrm{id}\right|_{\mathfrak{p}}$. A proof of this equivalence is given by Theorems 7.117 and 7.119 in [6].

Now assume $G / K$ is Hermitian, and let $z_{0}$ be an element of the center of $\mathfrak{k}$ such that $\left.\operatorname{ad}\left(z_{0}\right)\right|_{\mathfrak{p}} ^{2}=-\left.\mathrm{id}\right|_{\mathfrak{p}}$. It means that $\left.\operatorname{ad}\left(z_{0}\right)\right|_{\mathfrak{p}}$ defines a $K$-invariant $\mathbb{C}$-vector space structure on $\mathfrak{p}$. Denote by $\mathfrak{p}_{\mathbb{C}}$ the complexification of $\mathfrak{p}$, and similarly $\mathfrak{g}_{\mathbb{C}}$ and $\mathfrak{k}_{\mathbb{C}}$. The linear action of $K$ on $\mathfrak{p}$, defined by the adjoint action Ad, induces a complex-linear action of $K$ on $\mathfrak{p}_{\mathbb{C}}$.

Denote by $\mathfrak{p}^{ \pm, z_{0}}$ the eigenspace $\operatorname{ker}\left(\left.\operatorname{ad}\left(z_{0}\right)\right|_{\mathfrak{p}_{\mathbb{C}}} \mp i\right)$ of $\left.\operatorname{ad}\left(z_{0}\right)\right|_{\mathfrak{p}_{\mathbb{C}}}$ associated to the eigenvalue $\pm i$. Especially, $\operatorname{ad}\left(z_{0}\right)$ is multiplication by the complex number $\pm i$ on $\mathfrak{p}^{ \pm, z_{0}}$. These two subspaces of $\mathfrak{p}_{\mathbb{C}}$ are $K$-stable. 
Let $T$ be a maximal torus of the connected compact group $K$. We set the following convention: an element $\alpha \in \mathfrak{t}^{*}$ is a root of $\mathfrak{g}$ (resp. $\mathfrak{k}$ ) if there exists $X \in \mathfrak{g}_{\mathbb{C}}\left(\right.$ resp. $\left.X \in \mathfrak{k}_{\mathbb{C}}\right), X \neq 0$, such that $[H, X]=i \alpha(H) X$ for all $H \in \mathfrak{t}$. The associated root space is

$$
\mathfrak{g}_{\alpha}:=\left\{X \in \mathfrak{g}_{\mathbb{C}} \mid[H, X]=i \alpha(H) X, \forall H \in \mathfrak{t}\right\} .
$$

If $\alpha$ is a root of $\mathfrak{g}$, then either $\mathfrak{g}_{\alpha} \subseteq \mathfrak{k}_{\mathbb{C}}(\alpha$ is a compact root $)$, or $\mathfrak{g}_{\alpha} \subseteq \mathfrak{p}_{\mathbb{C}}$ (noncompact root). Note that the compact roots are the roots of the Lie algebra $\mathfrak{k}$. The set of compact (resp. noncompact) roots is denoted by $\mathfrak{R}_{c}\left(\right.$ resp. $\left.\mathfrak{R}_{n}\right)$. Fix once and for all $\mathfrak{t}_{+}^{*}$ as a Weyl chamber of $K$ in $\mathfrak{t}^{*}$, and let $\mathfrak{R}_{c}^{+}$be the system of positive compact roots associated to this Weyl chamber. Notice that, since $z_{0} \in \mathfrak{t}$, for any noncompact root $\beta$, we have either $\mathfrak{g}_{\beta} \subseteq \mathfrak{p}^{+, z_{0}}$ (positive noncompact roots) or $\mathfrak{g}_{\beta} \subseteq \mathfrak{p}^{-, z_{0}}$ (negative noncompact roots). Denote by $\mathfrak{R}_{n}^{+, z_{0}}$ the set of positive noncompact roots of $\mathfrak{g}$. Then $\mathfrak{R}_{c}^{+} \cup \mathfrak{R}_{n}^{+, z_{0}}$ is a system of positive roots of $\mathfrak{g}$. Indeed, we can easily see that for all $\alpha \in \mathfrak{R}_{c}^{+}$, we have $\alpha\left(z_{0}\right)=0$, and for all $\beta \in \mathfrak{R}_{n}^{+, z_{0}}, \beta\left(z_{0}\right)=1$.

Definition 2.1. The holomorphic chamber is the subchamber of $\mathfrak{t}_{+}^{*}$ defined by

$$
\mathcal{C}_{\text {hol }}^{z_{0}}:=\left\{\xi \in \mathfrak{t}^{*} \mid(\beta, \xi)>0, \forall \beta \in \mathfrak{R}_{n}^{+, z_{0}}\right\},
$$

where $(\cdot, \cdot)$ is the inner product on $\mathfrak{t}^{*}$ induced by $B_{\theta}$. A holomorphic coadjoint orbit is a coadjoint orbit $\mathcal{O}$ of $G$ which intersects $\mathcal{C}_{\text {hol }}^{z_{0}}$ in a nonempty set.

Let $\lambda \in \mathcal{C}_{\text {hol }}^{z_{0}}$. The holomorphic coadjoint orbit $G \cdot \lambda$ has a natural $G$-invariant Kähler structure:

1) a canonical $G$-invariant symplectic form $\Omega_{G \cdot \lambda}$, called the KirillovKostant-Souriau symplectic structure on $G \cdot \lambda$;

2) a $G$-invariant complex structure $J_{G \cdot \lambda}$ : its holomorphic tangent bundle $T^{1,0}(G \cdot \lambda) \rightarrow G \cdot \lambda$ is equal, above $\lambda$, to the $T$-submodule

$$
\sum_{\alpha \in \mathfrak{R}_{c}^{+},(\alpha, \lambda) \neq 0} \mathfrak{g}_{\alpha}+\underbrace{\sum_{\beta \in \mathfrak{R}_{n}^{-}} \mathfrak{g}_{\beta}}_{\mathfrak{p}^{-}}
$$

One can check that this complex structure is compatible with the symplectic form $\Omega_{G \cdot \lambda}$.

Besides, the stabilizer of $\lambda$ is clearly compact, since $(\beta, \lambda) \neq 0$ for all $\beta \in \mathfrak{R}_{n}$.

A trivial example of an element of $\mathcal{C}_{\text {hol }}^{z_{0}}$ is the linear form $\lambda_{0}:=$ $B_{\theta}\left(z_{0}, \cdot\right)$, since $\left(\beta, \lambda_{0}\right)=\beta\left(z_{0}\right)=1$ for all $\beta \in \mathfrak{R}_{n}^{+, z_{0}}$. This element is stabilized by the action of $K$.

REMARK 2.1. In the rest of the paper, we will omit the notation $z_{0}$ in $\mathcal{C}_{\text {hol }}^{z_{0}}$, and write $\mathcal{C}_{\text {hol }}$ instead. 
Example 2.1. Consider the example of $S U(p, q)$, with $1 \leq p \leq q$. A maximal compact subgroup is $K=S(U(p) \times U(q))$, and we fix for maximal torus the subgroup of diagonal matrices of $K$. The dual of its Lie algebra is $\mathfrak{t}^{*}=\left\{\left(x_{1}, \ldots, x_{p+q}\right) \in \mathbb{R}^{p+q} \mid \sum_{j} x_{j}=0\right\}$. For a standard choice of system of positive compact roots $\mathfrak{R}_{c}^{+}$, the Weyl chamber is

$$
\mathfrak{t}_{+}^{*}=\left\{\left(x_{1}, \ldots, x_{p+q}\right) \in \mathfrak{t}^{*} \mid x_{1} \geq \cdots \geq x_{p} \text { and } x_{p+1} \geq \cdots \geq x_{q}\right\} .
$$

We also choose for $z_{0}$ the matrix

$$
z_{0}:=\frac{i}{p+q}\left(\begin{array}{cc}
q I_{p} & 0 \\
0 & -p I_{q}
\end{array}\right) .
$$

The relative holomorphic chamber is

$$
\mathcal{C}_{\text {hol }}=\left\{\left(x_{1}, \ldots, x_{p+q}\right) \in \mathfrak{t}^{*} \mid x_{1} \geq \cdots \geq x_{p}>x_{p+1} \geq \cdots \geq x_{q}\right\} .
$$

The holomorphic coadjoint orbits of $S U(p, q)$ are the conjugation classes for $S U(p, q)$ of the diagonal matrices determined by the elements in $\mathcal{C}_{\text {hol }}$.

2.3. The symplectic forms $\Gamma^{*} \Omega_{G \cdot \lambda}$ and $\Omega_{K \cdot \lambda \times p}$. Let $\lambda \in \mathcal{C}_{\text {hol }}$. The manifold $K \cdot \lambda \times \mathfrak{p}$ can be identified with the holomorphic coadjoint orbit $G \cdot \lambda$ through the diffeomorphism $\Gamma$. This gives $K \cdot \lambda \times \mathfrak{p}$ a first canonical symplectic structure $\Gamma^{*} \Omega_{G \cdot \lambda}$, where $\Omega_{G \cdot \lambda}$ is the Kirillov-Kostant-Souriau symplectic form on $G \cdot \lambda$. Note that, here, $\Omega_{G \cdot \lambda}$ is defined by the formula

$$
\left.\left(\Omega_{G \cdot \lambda}\right)\right|_{g \lambda}([g,(X, A)],[g,(Y, B)])=\langle\lambda,[X, Y]\rangle+\langle\lambda,[A, B]\rangle
$$

for all $g \in G$, and all $(X, A),(Y, B) \in \mathfrak{k} / \mathfrak{k}_{\lambda} \oplus \mathfrak{p}$, where $\langle\cdot, \cdot\rangle: \mathfrak{g}^{*} \times \mathfrak{g} \rightarrow \mathbb{R}$ denotes the standard pairing. Then, from Lemma 2.1, the expression of $\Gamma^{*} \Omega_{G \cdot \lambda}$ is given for all $(k \lambda, Z) \in K \cdot \lambda \times \mathfrak{p}$ and all $(X, A),(Y, B) \in \mathfrak{k} / \mathfrak{k}_{\lambda} \oplus \mathfrak{p}$ by

$$
\begin{aligned}
\left.\left(\Gamma^{*} \Omega_{G \cdot \lambda}\right)\right|_{(k \lambda, Z)}(([k, X], A),([k, Y], B)) & \\
& =\left\langle\lambda,\left[X+\operatorname{Ad}\left(k^{-1}\right) \Psi_{Z}(A), Y+\operatorname{Ad}\left(k^{-1}\right) \Psi_{Z}(B)\right]\right\rangle .
\end{aligned}
$$

We can also split the right-hand term up using the operators $\Psi_{Z}^{+}$and $\Psi_{Z}^{-}$:

$$
\begin{aligned}
& \left.\left(\Gamma^{*} \Omega_{G \cdot \lambda}\right)\right|_{(k \lambda, Z)}(([k, X], A),([k, Y], B)) \\
& =\left\langle\lambda,\left[X+\operatorname{Ad}\left(k^{-1}\right) \Psi_{Z}^{-}(A), Y+\right.\right. \\
& \left.\left.\quad \operatorname{Ad}\left(k^{-1}\right) \Psi_{Z}^{-}(B)\right]\right\rangle \\
& +\left\langle k \lambda,\left[\Psi_{Z}^{+}(A), \Psi_{Z}^{+}(B)\right]\right\rangle .
\end{aligned}
$$

Now, consider the following $K$-invariant symplectic form on $\mathfrak{p}$ :

$$
\Omega_{\mathfrak{p}}(A, B):=\left\langle\lambda_{0},[A, B]\right\rangle=B_{\theta}\left(A, \operatorname{ad}\left(z_{0}\right) B\right), \quad \forall A, B \in \mathfrak{p} .
$$

It is symplectic since $\left.\operatorname{ad}\left(z_{0}\right)\right|_{\mathfrak{p}} ^{2}=-\mathrm{id}_{\mathfrak{p}}$ and $B_{\theta}$ is nondegenerate on $\mathfrak{p}$. The $K$-invariance of $\Omega_{\mathfrak{p}}$ is provided by the fact that $\lambda_{0}$ is centralized by $K$. Denoting $\Omega_{K \cdot \lambda}$ the Kirillov-Kostant-Souriau symplectic form on 
the compact coadjoint orbit $K \cdot \lambda$, we thus have another canonical $K$ invariant symplectic structure $\Omega_{K \cdot \lambda \times \mathfrak{p}}:=\Omega_{K \cdot \lambda} \oplus \Omega_{\mathfrak{p}}$, that is, the symplectic structure obtained from the direct product of the symplectic manifolds $\left(K \cdot \lambda, \Omega_{K \cdot \lambda}\right)$ and $\left(\mathfrak{p}, \Omega_{\mathfrak{p}}\right)$. This new symplectic form is given by the formula

$$
\left.\Omega_{K \cdot \lambda \times \mathfrak{p}}\right|_{(k \lambda, Z)}(([k, X], A),([k, Y], B))=\langle\lambda,[X, Y]\rangle+\left\langle\lambda_{0},[A, B]\right\rangle
$$

for all $(k \lambda, Z) \in K \cdot \lambda \times \mathfrak{p}$ and all $(X, A),(Y, B) \in \mathfrak{k} / \mathfrak{k}_{\lambda} \oplus \mathfrak{p}$.

Our purpose boils down to prove the existence of a symplectomorphism between the two symplectic manifolds $\left(K \cdot \lambda \times \mathfrak{p}, \Gamma^{*} \Omega_{G \cdot \lambda}\right)$ and $\left(K \cdot \lambda \times \mathfrak{p}, \Omega_{K \cdot \lambda \times \mathfrak{p}}\right)$.

\section{A noncompact version of Moser's theorem}

We first need a tool allowing us to prove that two symplectic manifolds are diffeomorphic. In general, Moser's trick [9] is an effective way of solving such a problem.

This trick is based on the following observation: if $\left(\Omega_{t}\right)_{t \in[0,1]}$ is a smooth family of symplectic forms on a compact manifold $N$, the existence of an isotopy $\rho_{t}: N \rightarrow N$ such that $\rho_{t}^{*} \Omega_{t}=\Omega_{0}$ for all $t \in[0,1]$ is equivalent to solving the equation $L_{\xi_{t}} \Omega_{t}+\frac{d}{d t} \Omega_{t}=0$ for the timedependent vector field $\xi_{t}$. The isotopy and the time-dependent vector field are related by the formula $\xi_{t}=\left(\frac{d}{d t} \rho_{t}\right) \circ \rho_{t}^{-1}$.

However, in the noncompact setting, it is not always possible to apply Moser's trick because the time-dependent vector field $\xi_{t}$ is not necessarily integrable. Fortunately, this is still possible in good cases. For instance, in [8], the proof uses the geodesic completeness of the manifold, combined with Rauch's comparison theorem. Another method is to work on Hamiltonian manifolds with proper moment maps, as in [5]. Here, we propose to follow this second method.

Let $K$ be a connected compact Lie group, $V$ a finite dimensional real representation of $K$, and $M$ a connected compact manifold, endowed with an action of $K$. This induces a diagonal action of $K$ on the trivial vector bundle $M \times V$. Let $\left(\Omega_{t}, \Phi_{t}\right)_{t \in[0,1]}$ be a smooth family of $K$-Hamiltonian structures on $M \times V$.

Theorem 3.1. Assume that the following assertions are satisfied:

1) there exists a smooth family $\left(\mu_{t}\right)_{t \in[0,1]}$ of $K$-invariant 1-forms such that $d \mu_{t}=\frac{d}{d t} \Omega_{t}$ for all $t \in[0,1]$;

2) for all $m \in M$ and all $t \in[0,1]$, we have an $\Omega_{t}$-orthogonal decomposition $T_{(m, 0)}(M \times V)=T_{m} M \oplus V$;

3) there exist two positive numbers $d$ and $\gamma$ such that

$$
\left\|\Phi_{t}(m, v)\right\| \geqslant d\|v\|^{\gamma}, \quad \forall(m, v) \in M \times V, \forall t \in[0,1] .
$$

Then there exists a K-invariant isotopy $\rho_{t}: M \times V \rightarrow M \times V$ such that $\rho_{t}^{*} \Omega_{t}=\Omega_{0}$ for all $t \in[0,1]$. 
Moreover, if, for some $m_{0} \in M$, we have $\left.\mu_{t}\right|_{\left(m_{0}, 0\right)}(u, 0)=0$ for all $(t, u) \in[0,1] \times T_{m_{0}} M$, then $\rho_{t}\left(m_{0}, 0\right)=\left(m_{0}, 0\right)$ for all $t \in[0,1]$.

The idea is to integrate the $K$-invariant time-dependent vector field $\xi_{t}$ defined on $M \times V$ by

$$
\imath\left(\xi_{t}\right) \Omega_{t}=-\mu_{t}, \quad \forall t \in[0,1] .
$$

Assertion 1) ensures that the isotopy $\rho_{t}$, obtained by integrating $\xi_{t}$, satisfies the equation $\rho_{t}^{*} \Omega_{t}=\Omega_{0}$. The other three conditions are used to make $\xi_{t}$ completely integrable on the noncompact manifold $M \times V$. Note that the last condition implies the properness of $\Phi_{t}$.

We are going to see that we can take $\mu_{t}$ so that the isotopy $\rho_{t}$ will satisfy $\rho_{t}^{*} \Phi_{t}=\Phi_{0}+c_{t}$, where $c_{t}$ is some element of $\mathfrak{k}^{*}$ depending only on $t$ (and not on the initial condition $x$ ).

Lemma 3.2. Let $\left(\mu_{t}\right)_{t \in[0,1]}$ be a smooth family of $K$-invariant 1 forms on $M \times V$. There exists a smooth family $\left(f_{t}\right)_{t \in[0,1]}$ of $K$-invariant $C^{\infty}$-functions on $M \times V$, such that

(i) $\left.d f_{t}\right|_{T(M \times\{0\})} \equiv 0$,

(ii) $\imath(v)\left(\mu_{t}-d f_{t}\right)=0$ on $M \times\{0\}$ for all $v \in V$.

Proof. Take $f_{t}(m, v):=\left.2 \int_{0}^{1} \mu_{t}\right|_{(m, s v)}(0, s v) d s$ for all $t \in[0,1]$ and all $(m, v) \in M \times V$.

q.e.d.

By Lemma 3.2, without loss of generality we may assume that, for all $t \in[0,1]$, the 1 -form $\mu_{t}$ is $K$-invariant and satisfies

$$
\left.\mu_{t}\right|_{(m, 0)}(0, v)=0, \quad \forall(m, v) \in M \times V .
$$

From (8) and (9), we notice that, for all $(m, v) \in M \times V$, the tangent vector $\xi_{t}(m, 0)$ is $\Omega_{t}$-orthogonal to $V$ at the point $(m, 0)$. But hypothesis 2 ) implies that $\xi_{t}(m, 0)$ is in $T_{m} M$ for all $m \in M$ and all $t \in[0,1]$.

In order to prove Theorem 3.1, we must integrate the smooth family of vector fields $\xi_{t}$ and obtain an isotopy on $M \times V$. We thus have to consider the time-dependent differential equation

$$
\left\{\begin{array}{l}
\rho_{0}(x)=x \\
\frac{d}{d t} \rho_{t}(x)=\xi_{t}\left(\rho_{t}(x)\right)
\end{array}\right.
$$

for any initial condition $x \in M \times V$.

We will need the following properties of the integration of a smooth family of vector fields with initial conditions contained in a relatively compact set.

Proposition 3.3. Let $\xi_{t}$ be a smooth family of vector fields on a manifold $N$, and $U$ a relatively compact set in $N$.

(i) There exists $\varepsilon \in] 0,1]$ and a map $\rho^{\varepsilon, U}:[0, \varepsilon[\times U \rightarrow N$ which satisfies the differential equation (10). 
(ii) If Image $\left(\rho^{\varepsilon, U}\right)$ is relatively compact in $N$, then we can extend $\rho^{\varepsilon, U}$ in $\rho^{\varepsilon^{\prime}, U}$ with $\varepsilon^{\prime}>\varepsilon$.

Proof. By Cauchy-Lipschitz's theorem, the domain of definition $\mathcal{D} \subseteq$ $[0,1] \times N$ of the integral curves of (10) is an open set. Thus, for any $x \in U$, since the domain $\mathcal{D}$ contains $(0, x) \in[0,1] \times N$, there exists an open neighborhood $\mathscr{V}_{x}$ of $x$ in $N$ and a real number $\left.\left.\varepsilon_{x} \in\right] 0,1\right]$ such that $\left[0, \varepsilon_{x}[\times \mathscr{V} \subseteq \mathcal{D}\right.$. But $U$ is relatively compact, so there exists a finite number of points $x_{1}, \ldots, x_{s}$ in $U$ such that the family of neighborhoods $\left(\mathscr{V}_{x_{j}}\right)_{j=1, \ldots, s}$ is a covering of $U$. Take $\varepsilon:=\min _{j=1}^{s} \varepsilon_{x_{j}}$. Then, clearly $[0, \varepsilon[\times U$ is contained in $\mathcal{D}$. This proves assertion $(i)$.

Now assume that Image $\left(\rho^{\varepsilon, U}\right)$ is relatively compact in $N$. In particular, any integral curve $\rho^{\varepsilon, U}(\cdot, x)$, with $x \in U$, is contained in a compact set, so we can extend it to an integral curve of (10) defined on all $[0, \varepsilon]$. That is, $[0, \varepsilon] \times U \subseteq \mathcal{D}$. Applying the same argument as in the previous paragraph for $(\varepsilon, x)$ instead of $(0, x)$, we show that there exists $\varepsilon^{\prime}>\varepsilon$ such that $\left[0, \varepsilon^{\prime}[\times U \subseteq \mathcal{D}\right.$ and prove assertion $(i i)$ q.e.d.

Proof of Theorem 3.1. Let $r$ be any positive real number, and $U_{r}:=$ $M \times B(0, r)$ the open connected neighborhood of $M \times\{0\}$ in $M \times V$, where $B(0, r)$ is the open ball in $V$ centered at 0 with radius $r$, defined for some $K$-invariant inner product on $V$. The closure $\bar{U}_{r}$ is the compact subset $M \times \bar{B}(0, r)$ of $M \times V$.

First, we have to prove two claims.

Claim 1. For all $m \in M$, the integral curve $t \mapsto \rho_{t}(m, 0)$ is defined for all $t \in[0,1]$.

Since $\xi_{t}(m, 0)$ is tangent to the submanifold $M \times\{0\}$ for all $m \in M$ and $t \in[0,1]$, the integral curve $t \mapsto \rho_{t}(m, 0)$ is included in $M \times\{0\}$ for all $m \in M$. But $M$ is compact. Hence, the maximal integral curve of (10), starting from any point $(m, 0) \in M \times\{0\}$, is defined for all $t \in[0,1]$.

Let $m_{0}$ be an element of $M$. This point enables us to define the family of vectors

$$
c_{t}:=\Phi_{t} \circ \rho_{t}\left(m_{0}, 0\right)-\Phi_{0}\left(m_{0}, 0\right) \in \mathfrak{k}^{*}, \quad \forall t \in[0,1],
$$

and the constant

$$
C:=\sup _{t \in[0,1]}\left\|c_{t}\right\|<+\infty .
$$

This supremum is finite because the family of moment maps $\left(\Phi_{t}\right)_{t \in[0,1]}$ is smooth. We also define the real numbers

$$
D_{r}:=\sup _{x \in \bar{U}_{r}}\left\|\Phi_{0}(x)\right\|, \quad \forall r>0 .
$$


Claim 2. Let $r>0$ and $\varepsilon \in] 0,1]$ be such that the map $\rho^{\varepsilon, U_{r}}$ : $\left[0, \varepsilon\left[\times U_{r} \rightarrow M \times V\right.\right.$ is defined, as in Proposition 3.3. Then we have

$$
\text { Image }\left(\rho^{\varepsilon, U_{r}}\right) \subset \bar{U}_{\left(\frac{D_{r}+C}{d}\right)^{1 / \gamma}}
$$

For $r>0$ and $\varepsilon \in] 0,1]$ satisfying the hypothesis of Claim 2, we obtain for all $t \in\left[0, \varepsilon\left[\right.\right.$ a smooth map $\rho_{t}^{\varepsilon, U_{r}}:=\rho^{\varepsilon, U_{r}}(t, \cdot): U_{r} \rightarrow M \times V$. Since $\Omega_{t}$ is a closed 2-form, $L_{\xi_{t}} \Omega_{t}=d\left(\imath\left(\xi_{t}\right) \Omega_{t}\right)=-d \mu_{t}=-\frac{d}{d t} \Omega_{t}$ by Cartan's Formula. Thus, $0=\left(\rho_{t}^{\varepsilon, U_{r}}\right)^{*}\left(L_{\xi_{t}} \Omega_{t}+\frac{d}{d t} \Omega_{t}\right)=\frac{d}{d t}\left(\left(\rho_{t}^{\varepsilon, U_{r}}\right)^{*} \Omega_{t}\right)$ on $U_{r}$, for all $t \in[0, \varepsilon[$. As a result, we have

$$
\left(\rho_{t}^{\varepsilon, U_{r}}\right)^{*} \Omega_{t}=\left.\Omega_{0}\right|_{U_{r}}, \quad \forall t \in[0, \varepsilon[.
$$

Note that $U_{r}$ is a $K$-invariant neighborhood of $M \times\{0\}$ in $M \times V$. Therefore, $\left.\Phi_{0}\right|_{U_{r}}: U_{r} \rightarrow \mathfrak{k}^{*}$ is a moment map for the symplectic $K$ manifold $\left(U_{r},\left.\Omega_{0}\right|_{U_{r}}\right)$. But, by $(12),\left(\rho_{t}^{\varepsilon, U_{r}}\right)^{*} \Phi_{t}=\Phi_{t} \circ \rho_{t}^{\varepsilon, U_{r}}: U_{r} \rightarrow \mathfrak{k}^{*}$ is another moment map of $\left(U_{r},\left.\Omega_{0}\right|_{U_{r}}\right)$. We deduce from the connectedness of $U_{r}$ that, for all $t \in\left[0, \varepsilon\left[, \Phi_{t} \circ \rho_{t}^{\varepsilon, U_{r}}-\Phi_{0}\right.\right.$ is a constant map and, more precisely, we have the equality $\Phi_{t} \circ \rho_{t}^{\varepsilon, U_{r}}(x)=\Phi_{0}(x)+c_{t}$ for all $x \in U_{r}$ and all $t \in[0, \varepsilon[$. In particular, this induces the inequality

$$
\left\|\Phi_{t}\left(\rho_{t}^{\varepsilon, U_{r}}(x)\right)\right\| \leq\left\|\Phi_{0}(x)\right\|+\left\|c_{t}\right\| \leq D_{r}+C, \quad \forall x \in U_{r}, \forall t \in[0, \varepsilon[.
$$

Denote by $\pi_{V}: M \times V \rightarrow V$ the canonical projection. From hypothesis $3)$ and (13), we have

$$
D_{r}+C \geq\left\|\Phi_{t}\left(\rho_{t}^{\varepsilon, U_{r}}(x)\right)\right\| \geq d\left\|\pi_{V}\left(\rho_{t}^{\varepsilon, U_{r}}(x)\right)\right\|^{\gamma}, \quad \forall x \in U_{r}, \forall t \in[0, \varepsilon[,
$$

that is, the inclusion $\operatorname{Image}\left(\rho_{t}^{\varepsilon, U_{r}}\right) \subseteq \bar{U}_{\left(\frac{D_{r}+C}{d}\right)^{1 / \gamma}}$.

Now, for all $r>0$, we define the interval

$$
I_{r}:=\left\{\varepsilon \in[0,1] \mid\left[0, \varepsilon\left[\times U_{r} \subseteq \mathcal{D}\right\} \subseteq[0,1] .\right.\right.
$$

We recall that, here, $\mathcal{D} \subseteq[0,1] \times M \times V$ is the domain of definition of the integral curves of the time-dependent differential equation (10).

We want to prove that $I_{r}$ is equal to $[0,1]$ for all $r$. By Proposition $3.3(i), I_{r}$ contains a nonzero element. Moreover, Proposition 3.3 (ii) and Claim 2 prove that $I_{r}$ is open in $[0,1]$.

Let $\varepsilon_{r}:=\sup I_{r}$. By property of the supremum, $\left[0, \varepsilon\left[\times U_{r}\right.\right.$ must be contained in the domain $\mathcal{D}$ of (10) for all $\varepsilon<\varepsilon_{r}$. Hence, $\left[0, \varepsilon_{r}\left[\times U_{r}\right.\right.$ is clearly contained in $\mathcal{D}$, that is, $\varepsilon_{r} \in I_{r}$ and $I_{r}$ is a closed interval.

Finally, since $I_{r}$ is nonempty, open, and closed in the connected set $[0,1]$, we have $I_{r}=[0,1]$, and, thus, every integral curve with initial condition in $U_{r}$ is completely integrable. Therefore, the time-dependent vector field $\xi_{t}$ is complete in $M \times V=\cup_{r>0} U_{r}$. It defines, for all $t \in[0,1]$, a map $\rho_{t}: M \times V \mapsto M \times V$, which is a diffeomorphism onto [7, Theorem 52 ], and $K$-equivariant because $\xi_{t}$ is $K$-invariant. This proves that $\rho_{t}$ is 
a $K$-equivariant isotopy of $M \times V$ that satisfies the equality $\rho_{t}^{*} \Omega_{t}=\Omega_{0}$ for all $t \in[0,1]$.

It remains to prove the last assertion. We assumed, at the beginning of the proof, that $\left(\mu_{t}\right)_{t \in[0,1]}$ is a smooth family of $K$-invariant 1-forms such that $\left.\mu_{t}\right|_{(m, 0)}(0, v)=0$ for all $(m, v) \in M \times V$. But now, the hypothesis on $m_{0}$ yields $\left.\mu_{t}\right|_{\left(m_{0}, 0\right)} \equiv 0$, and then $\xi_{t}\left(m_{0}, 0\right)=0$, for all $t \in[0,1]$. By uniqueness of the maximal integral curve of $\xi_{t}$ with initial condition $\left(m_{0}, 0\right)$, we conclude that $\rho_{t}\left(m_{0}, 0\right)=\left(m_{0}, 0\right)$ for all $t \in[0,1]$. This completes the theorem's proof.

q.e.d.

Theorem 3.1 works for every smooth family $\left(\Omega_{t}\right)_{t \in[0,1]}$ of symplectic forms, not only for segments as in the classical Moser argument. But it is generally difficult to find moment maps for arbitrary symplectic paths. So, for practical purposes, we study segments of symplectic forms as soon as possible.

Note that the manifold we are studying here is the trivial vector bundle $M \times V$. Thus we can define the two canonical maps

$$
i: M \hookrightarrow M \times V \quad \text { and } \quad \pi_{M}: M \times V \rightarrow M .
$$

The map

$$
F: M \times V \times[0,1] \rightarrow M \times V,(m, v, t) \mapsto(m, t v)
$$

is a $K$-equivariant homotopy of $M \times V$ such that $F(m, v, 0)=i$ 。 $\pi_{M}(m, v)=(m, 0)$ and $F(m, v, 1)=\operatorname{id}_{M \times V}(m, v)$. For any 2-form $\omega$ we define the 1 -form $h_{F}(\omega)$ at $(m, v) \in M \times V$ by the formula $\left.h_{F}(\omega)\right|_{(m, v)}:=\left.\int_{t=0}^{t=1}\left(F^{*} \omega\right)\right|_{(m, v, t)}$. By the Poincaré Lemma, we have

$$
d \circ h_{F}+h_{F} \circ d=\operatorname{id}_{M \times V}^{*}-\pi_{M}^{*} \circ i^{*}
$$

(see for example [12]). One can easily check from the definition that, if $\omega$ is $K$-invariant, then $h_{F}(\omega)$ is. Moreover, the 1 -form $h_{F}(\omega)$ vanishes on the submanifold $M \times\{0\}$.

Corollary 3.4. Let $\Omega_{0}$ and $\Omega_{1}$ be two $K$-invariant symplectic forms on $M \times V$, with moment maps $\Phi_{0}$ and $\Phi_{1}$ respectively. Set $\Omega_{t}=t \Omega_{1}+$ $(1-t) \Omega_{0}$ and $\Phi_{t}=t \Phi_{1}+(1-t) \Phi_{0}$ for all $t \in[0,1]$. If the following assertions are satisfied:

a) for all $t \in[0,1], \Omega_{t}$ is symplectic on $M \times V$,

b) $i^{*} \Omega_{0}=i^{*} \Omega_{1}$ on $M$,

c) for all $m \in M$, the decomposition $T_{(m, 0)}(M \times V)=T_{m} M \oplus V$ is orthogonal for $\Omega_{0}$ and $\Omega_{1}$,

d) there exist two positive numbers $d$ and $\gamma$ such that

$$
\left\|\Phi_{t}(m, v)\right\| \geqslant d\|v\|^{\gamma}, \quad \forall(m, v) \in M \times V, \forall t \in[0,1],
$$

then, there exists a $K$-equivariant symplectomorphism from $\left(M \times V, \Omega_{0}\right)$ onto $\left(M \times V, \Omega_{1}\right)$ fixing each element of $M \times\{0\}$. 
Proof. Let $\mu$ be the 1 -form defined by $\mu=h_{F}\left(\Omega_{1}-\Omega_{0}\right)$. Hypothesis b) and formula (14) yield that $d \mu=\Omega_{1}-\Omega_{0}$, because $\Omega_{1}-\Omega_{0}$ is a closed 2-form and it must be in the kernel of the linear operator $i^{*}$. Thus assertion 1) of Theorem 3.1 is satisfied. From the definition of $\Omega_{t}$, the decomposition in hypothesis c) is also $\Omega_{t}$-orthogonal. Now assertion 2) is verified, so we can conclude the proof, applying Theorem 3.1 with $\mu=h_{F}\left(\Omega_{1}-\Omega_{0}\right)$, which vanishes on $M \times\{0\}$, so that we get a symplectomorphism fixing each point of $M \times\{0\}$.

q.e.d.

Example 3.1. Assume that $\left(V, \omega^{1}\right)$ is a Hamiltonian $K$-manifold, with moment map $\Phi^{1}$ such that there exist two positive real numbers $d$ and $\gamma$ satisfying the assertion:

$$
\left\|\Phi^{1}(v)\right\| \geq d\|v\|^{\gamma}, \quad \forall v \in V .
$$

Let $\delta$ be a positive real number, and $\omega^{\delta}:=\delta \omega^{1}$. For every $t \in[0,1]$, the 2-form $\omega_{t}:=t \omega^{1}+(1-t) \omega^{\delta}=(t+(1-t) \delta) \omega^{1}$ is symplectic, and $\Phi_{t}:=(t+(1-t) \delta) \Phi^{1}$ is a moment map for the Hamiltonian $K$-manifold $\left(V, \omega_{t}\right)$. Since $M$ is a single point here, hypotheses a), b), and c) of Corollary 3.4 are clearly satisfied, and assertion d) is given by

$$
\left\|\Phi_{t}(v)\right\|=(t+(1-t) \delta)\left\|\Phi^{1}(v)\right\| \geq \min \{1, \delta\} d\|v\|^{\gamma}, \quad \forall v \in V .
$$

Therefore, there exists a $K$-equivariant diffeomorphism from $V$ onto $V$ which takes $\omega^{1}$ to $\omega^{\delta}$.

\section{Proof of the Hermitian symmetric space case}

In this section, we prove Theorem 1.1 when the holomorphic coadjoint orbit is $G \cdot \lambda_{0}$, where $\lambda_{0}=B_{\theta}\left(z_{0}, \cdot\right)$ is the element of $\mathfrak{g}^{*}$ identified with $z_{0}$ using the inner product $B_{\theta}$ on $\mathfrak{g}$. The Hermitian symmetric space $G / K$ coincides with the coadjoint orbit $G \cdot \lambda_{0}$ since $\lambda_{0}$ is centralized by $K$. The diffeomorphism $\Gamma$ is expressed here by the map

$$
\begin{aligned}
\Gamma_{0}: \mathfrak{p} & \longrightarrow G \cdot \lambda_{0} \\
Z & \longmapsto e^{Z} \lambda_{0} .
\end{aligned}
$$

The symplectic form $\Gamma_{0}^{*} \Omega_{G \cdot \lambda_{0}}$, given by the formula (5) in the general case, is now

$$
\left.\left(\Gamma_{0}^{*} \Omega_{G \cdot \lambda_{0}}\right)\right|_{Z}(A, B)=\left\langle\lambda_{0},\left[\Psi_{Z}^{+}(A), \Psi_{Z}^{+}(B)\right]\right\rangle, \quad \forall A, B \in \mathfrak{p} .
$$

Theorem 4.1 (McDuff). There exists a $K$-equivariant diffeomorphism from manifold $G \cdot \lambda_{0}$ onto $\mathfrak{p}$ which takes the symplectic form $\Omega_{G \cdot \lambda_{0}}$ on $G \cdot \lambda_{0}$ to the symplectic form $\Omega_{\mathfrak{p}}$ on $\mathfrak{p}$, such that $\lambda_{0} \in G \cdot \lambda_{0}$ is sent to $0 \in \mathfrak{p}$.

We present here a completely different proof of this result, using Theorem 3.1. The main difference is that we need proper moment maps on our Hamiltonian $K$-manifolds. 
The canonical projection map $G \cdot \lambda_{0} \subset \mathfrak{g}^{*} \rightarrow \mathfrak{k}^{*}$ is known to be a moment map of the Hamiltonian $K$-manifold $\left(G \cdot \lambda_{0}, \Omega_{G \cdot \lambda_{0}}\right)$. Composing with $\Gamma_{0}$, we get a moment map $\Phi_{\Gamma_{0}^{*} \Omega_{G \cdot \lambda_{0}}}$ for the Hamiltonian $K$-manifold $\left(\mathfrak{p}, \Gamma_{0}^{*} \Omega_{G \cdot \lambda_{0}}\right)$. This moment map is defined by

$$
\begin{aligned}
\Phi_{\Gamma_{0}^{*} \Omega_{G \cdot \lambda_{0}}}: \mathfrak{p} & \rightarrow \mathfrak{k}^{*} \\
Z & \mapsto\left(X \in \mathfrak{k} \mapsto\left\langle e^{Z} \lambda_{0}, X\right\rangle\right) .
\end{aligned}
$$

Lemma 4.2. For all $Z \in \mathfrak{p}$, we have

$$
\left\langle\Phi_{\Gamma_{0}^{*} \Omega_{G \cdot \lambda_{0}}}(Z)-\lambda_{0}, z_{0}\right\rangle \geqslant \frac{1}{2}\|Z\|^{2} .
$$

In particular, the moment map $\Phi_{\Gamma_{0}^{*} \Omega_{G \cdot \lambda_{0}}}: \mathfrak{p} \rightarrow \mathfrak{k}^{*}$ is proper.

Proof. First notice that, for all $Z \in \mathfrak{p}$ and all $X \in \mathfrak{k}$, we have

$$
\left\langle\lambda_{0}, e^{-\operatorname{ad}(Z)} X\right\rangle=\left\langle\lambda_{0}, \sum_{k=0}^{\infty} \frac{\operatorname{ad}(-Z)^{2 k}}{(2 k) !} X\right\rangle=B_{\theta}\left(z_{0}, \sum_{k=0}^{\infty} \frac{\operatorname{ad}(Z)^{2 k}}{(2 k) !} X\right) \text {. }
$$

$\operatorname{But} \operatorname{ad}(Z)$ is symmetric for the inner product $B_{\theta}$. Thus, we get

$$
\begin{aligned}
\left\langle e^{Z} \lambda_{0}-\lambda_{0}, z_{0}\right\rangle & =\sum_{k=1}^{\infty} \frac{1}{(2 k) !} B_{\theta}\left(\operatorname{ad}(Z)^{k} z_{0}, \operatorname{ad}(Z)^{k} z_{0}\right) \\
& \geqslant \frac{1}{2} B_{\theta}\left(\left[z_{0}, Z\right],\left[z_{0}, Z\right]\right)=\frac{1}{2}\|Z\|^{2} .
\end{aligned}
$$

Consequently, the map $\Phi_{\Gamma_{0}^{*} \Omega_{G \cdot \lambda_{0}}}-\lambda_{0}: \mathfrak{p} \rightarrow \mathfrak{k}^{*}$ is proper, and so is $\Phi_{\Gamma_{0}^{*} \Omega_{G \cdot \lambda_{0}}}$.

q.e.d.

As for the second symplectic form $\Omega_{\mathfrak{p}}$ on $\mathfrak{p}$, it is a constant symplectic form on a symplectic vector space. Recall that it is defined by (6). Therefore, one can easily check that a moment map for $\left(\mathfrak{p}, \Omega_{\mathfrak{p}}\right)$ is

$$
\begin{aligned}
\Phi_{\Omega_{\mathfrak{p}}}: \mathfrak{p} & \rightarrow \mathfrak{k}^{*} \\
Z & \mapsto\left(X \in \mathfrak{k} \mapsto \frac{1}{2}\left\langle\lambda_{0},[[X, Z], Z]\right\rangle\right) .
\end{aligned}
$$

Lemma 4.3. We have $\left\langle\Phi_{\Omega_{\mathfrak{p}}}(Z), z_{0}\right\rangle=\frac{1}{2}\|Z\|^{2}$, for all $Z \in \mathfrak{p}$. In particular, the moment map $\Phi_{\Omega_{\mathfrak{p}}}: \mathfrak{p} \rightarrow \mathfrak{k}^{*}$ is proper.

Proof. From the definition of $\Phi_{\Omega_{\mathfrak{p}}}$, we obtain the following equalities:

$$
\left\langle\Phi_{\Omega_{\mathfrak{p}}}(Z), z_{0}\right\rangle=\frac{1}{2} B_{\theta}\left(z_{0},\left[\left[z_{0}, Z\right], Z\right]\right)=\frac{1}{2} B_{\theta}\left(-\operatorname{ad}\left(z_{0}\right)^{2} Z, Z\right) .
$$

$\left.\operatorname{But} \operatorname{ad}\left(z_{0}\right)\right|_{\mathfrak{p}} ^{2}=-\mathrm{id}_{\mathfrak{p}}$. Thus $\left\langle\Phi_{\Omega_{\mathfrak{p}}}(Z), z_{0}\right\rangle=\frac{1}{2}\|Z\|^{2}$.

q.e.d.

We will also need the next lemma, which is an analogue of the Poincaré Lemma for smooth families of differential forms.

Lemma 4.4. Let $\left(\omega_{t}\right)_{t \in[0,1]}$ be a smooth family of closed 2-forms on $\mathfrak{p}$. Then there exists a smooth family $\left(\mu_{t}\right)_{t \in[0,1]}$ of 1 -forms, such that $\omega_{t}=d \mu_{t}$ for all $t \in[0,1]$. Moreover, if the 2 -form $\omega_{t}$ is $K$-invariant for $t \in[0,1]$, then we can take $\mu_{t}$ to be $K$-invariant. 
The proof of this lemma is almost the same as the one of the Poincaré Lemma for 2 -forms on $\mathbb{R}^{n}$ (see for example [12, 4.18]), by making obvious changes of notation. The parameter $t$ does not involve any change in the computations, and the result is actually a smooth family.

Moreover, if $\omega_{t}$ is $K$-invariant, then the linearity of the action of $K$ on $\mathfrak{p}$ induces that $\mu_{t}$ is $K$-invariant. This can be checked directly on the definition of $\mu_{t}$ given in the proof of the Poincaré Lemma.

Proof of Theorem 4.1. For all $t \in[0,1]$, let $\Omega_{t}$ be the differential 2 -form on $\mathfrak{p}$ defined by

$$
\left.\Omega_{t}\right|_{Z}:=\left.\left(\Gamma_{0}^{*} \Omega_{G \cdot \lambda_{0}}\right)\right|_{t Z} \quad \forall Z \in \mathfrak{p} .
$$

In particular, $\Omega_{1}=\Gamma_{0}^{*} \Omega_{G \cdot \lambda_{0}}$. Moreover, for $t=0$, we have the constant symplectic form

$$
\left.\Omega_{0}\right|_{Z}(A, B)=\left\langle\lambda_{0},[A, B]\right\rangle=\left.\Omega_{\mathfrak{p}}\right|_{Z}(A, B),
$$

for all $Z, A, B \in \mathfrak{p}$, since $\Psi_{0}^{+}=\mathrm{id}_{\mathfrak{p}}$. When $t \neq 0$, one can check that

$$
\Omega_{t}=\frac{1}{t^{2}} \eta_{t}^{*}\left(\Gamma_{0}^{*} \Omega_{G \cdot \lambda_{0}}\right)=\frac{1}{t^{2}} \eta_{t}^{*} \Omega_{1}
$$

where $\eta_{t}: \mathfrak{p} \rightarrow \mathfrak{p}$ is the homothety $Z \mapsto t Z$, for all $t \in[0,1]$. By linearity of the action of $K, \eta_{t}$ commutes with this action. Thus $\Omega_{t}$ is $K$ invariant. Furthermore, since $\Omega_{1}$ is closed, we have $d \Omega_{t}=\frac{1}{t^{2}} d\left(\eta_{t}^{*} \Omega_{1}\right)=$ $\frac{1}{t^{2}} \eta_{t}^{*} d \Omega_{1}=0$. But $\Omega_{1}$ is symplectic, so the skew-symmetric bilinear form $\left.\left(\Omega_{t}\right)\right|_{Z}=\left.\left(\Omega_{1}\right)\right|_{t Z}$ is clearly nondegenerate. We conclude that $\Omega_{t}$ is symplectic for all $t \in[0,1]$.

The smooth family $\left(\Omega_{t}\right)_{t \in[0,1]}$ of symplectic forms induces the smooth family $\left(\frac{d}{d t} \Omega_{t}\right)_{t \in[0,1]}$ of $K$-invariant closed 2 -forms. Indeed, $\frac{d}{d t} \Omega_{t}$ is closed for all $t \in[0,1]$ since the exterior derivative $d$ and the differential operator $\frac{d}{d t}$ commute. Now, from Lemma 4.4, there exists a smooth family $\left(\mu_{t}\right)_{t \in[0,1]}$ of $K$-invariant 1 -forms on $\mathfrak{p}$ such that, for all $t \in[0,1]$, we have $\frac{d}{d t} \Omega_{t}=d \mu_{t}$. This proves hypothesis 1 ) of Theorem 3.1.

For all $t \in] 0,1]$, we define

$$
\Phi_{t}:=\frac{1}{t^{2}} \eta_{t}^{*} \Phi_{\Gamma_{0}^{*} \Omega_{G \cdot \lambda_{0}}}-\frac{1}{t^{2}} \lambda_{0}: \mathfrak{p} \rightarrow \mathfrak{k}^{*},
$$

and, for $t=0$, we set $\Phi_{0}:=\Phi_{\Omega_{\mathfrak{p}}}$. The maps $\Phi_{t}$ are moment maps for the Hamiltonian $K$-manifolds $\left(\mathfrak{p}, \Omega_{t}\right)$, since $\lambda_{0}$ is centralized by $K$. Notice that, for all $t \in[0,1]$,

$$
\left\langle\Phi_{t}(Z), X\right\rangle=\sum_{k=0}^{\infty} \frac{t^{2 k}}{(2 k+2) !} B_{\theta}\left(z_{0}, \operatorname{ad}(Z)^{2 k+2} X\right), \quad \forall X \in \mathfrak{k},
$$

so clearly the family $\left(\Phi_{t}\right)_{t \in[0,1]}$ is smooth.

The submanifold $M \times\{0\}=K \cdot \lambda_{0} \times\{0\}$ being identified to $\{0\} \subset \mathfrak{p}$, we have $\left(T_{0} \mathfrak{p}\right)^{\left.\Omega_{t}\right|_{0}}=\{0\}=T_{0}\{0\}$, that is, condition 2) is also satisfied. 
It remains to prove hypothesis 3) of Theorem 3.1. Formula (16) gives

$$
\left\langle\Phi_{t}(Z), z_{0}\right\rangle \geqslant \frac{1}{2}\|Z\|^{2}
$$

for all $Z \in \mathfrak{p}$ and all $t \in[0,1]$, similarly to the proof of Lemma 4.2. So $\left\|\Phi_{t}(Z)\right\|=\sup _{X \in \mathfrak{k} \backslash\{0\}} \frac{\left\langle\Phi_{t}(Z), X\right\rangle}{\|X\|} \geq \frac{1}{2\left\|z_{0}\right\|}\|Z\|^{2}$, for all $Z \in \mathfrak{p}$ and all $t \in[0,1]$. Finally assertion 4$)$ is proved and we conclude by applying Theorem 3.1 and the fact that the condition " $\left.\mu_{t}\right|_{0}(0)=0$ for all $t \in$ $[0,1]$ " is always satisfied on the vector space $\mathfrak{p} \simeq K \cdot \lambda_{0} \times \mathfrak{p}$. $\quad$ q.e.d.

\section{Proof of Theorem 1.1}

In this last section, we prove Theorem 1.1 for any $\lambda \in \mathcal{C}_{\text {hol }}$. Now, the two $K$-equivariant diffeomorphisms $\Gamma: K \cdot \lambda \times \mathfrak{p} \rightarrow G \cdot \lambda$ and $\Gamma_{0}: \mathfrak{p} \rightarrow G \cdot \lambda_{0}$ are involved, so that we will exclusively work on the manifold $K \cdot \lambda \times \mathfrak{p}$. We will consider the following symplectic forms on $K \cdot \lambda \times \mathfrak{p}:$

(i) $\Omega_{K \cdot \lambda \times \mathfrak{p}}=\Omega_{K \cdot \lambda} \oplus \Omega_{\mathfrak{p}}$;

(ii) $\Omega^{1}:=\Omega_{K \cdot \lambda} \oplus \Gamma_{0}^{*} \Omega_{G \cdot \lambda_{0}}$;

(iii) $\Omega^{\delta}:=\Omega_{K \cdot \lambda} \oplus\left(\delta \Gamma_{0}^{*} \Omega_{G \cdot \lambda_{0}}\right)$, for all $\delta>0$;

(iv) $\Gamma^{*} \Omega_{G \cdot \lambda}$.

Recall that the "direct sum" of two symplectic forms is defined as the canonical symplectic form on the direct product of the two underlying symplectic manifolds.

The purpose of this section is to prove that the symplectic forms $\Omega_{K \cdot \lambda \times \mathfrak{p}}$ and $\Gamma^{*} \Omega_{G \cdot \lambda}$ are symplectomorphic. To this end, we will use repeatedly the Moser argument given in section 3 in order to prove the existence of the symplectomorphisms indicated in the following diagram:

$$
\Omega_{K \cdot \lambda \times \mathfrak{p}} \stackrel{\text { Theorem 4.1 }}{\longrightarrow} \Omega^{1} \stackrel{\text { Example } 3.1}{\longrightarrow} \Omega^{\delta} \stackrel{\text { section } 5.1}{\longrightarrow} \Gamma^{*} \Omega_{G \cdot \lambda} .
$$

The first symplectomorphism directly results from Theorem 4.1, the second one from Example 3.1 and Lemma 4.2. The last arrow will be studied in the next subsection. Furthermore, one can assume that the diffeomorphisms, given by the three arrows in the above diagram, fix each point of the submanifold $K \cdot \lambda \times\{0\}$. Composing such diffeomorphisms yields a symplectomorphism from $\left(K \cdot \lambda \times \mathfrak{p}, \Omega_{K \cdot \lambda \times \mathfrak{p}}\right)$ onto $\left(K \cdot \lambda \times \mathfrak{p}, \Gamma^{*} \Omega_{G \cdot \lambda}\right)$, which satisfies the statement of Theorem 1.1.

5.1. Symplectomorphism between $\Omega^{\delta}$ and $\Gamma^{*} \Omega_{G \cdot \lambda}$ on $K \cdot \lambda \times \mathfrak{p}$. The proof of Theorem 1.1 will be completed by proving the next statement.

Theorem 5.1. For all $\delta>b_{\lambda}:=\sup _{\|u\|=1,\|v\|=1}\langle\lambda,[u, v]\rangle$, there exists a $K$-equivariant symplectomorphism from $\left(K \cdot \lambda \times \mathfrak{p}, \Gamma^{*} \Omega_{G \cdot \lambda}\right)$ onto $(K$. $\left.\lambda \times \mathfrak{p}, \Omega^{\delta}\right)$, which fixes each point $(k \lambda, 0)$, for all $k \in K$. 
We will apply Corollary 3.4 again. The main difficulty lies in proving that the 2-form $\Omega_{t}^{\delta}:=t \Gamma^{*} \Omega_{G \cdot \lambda}+(1-t) \Omega^{\delta}$ is symplectic for all $t \in[0,1]$. According to the statement of the next theorem, this is possible for $\delta$ large enough.

We first define a Hamiltonian structure on $K \cdot \lambda \times \mathfrak{p}$ compatible with $\Omega_{t}^{\delta}$. A moment map for the Hamiltonian $K$-manifold $\left(K \cdot \lambda \times \mathfrak{p}, \Gamma^{*} \Omega_{G \cdot \lambda}\right)$ is the map defined for all $(k \lambda, Z) \in K \cdot \lambda \times \mathfrak{p}$ by

$$
\Phi_{\Gamma^{*} \Omega_{G \cdot \lambda}}(k \lambda, Z):=\left.\left(e^{Z} k \lambda\right)\right|_{\mathfrak{k}}=k \lambda \circ\left(\sum_{n \geqslant 0} \frac{\operatorname{ad}(Z)^{2 n}}{(2 n) !}\right) .
$$

Obviously, the map $\Phi^{\delta}$, defined for all $(k \lambda, Z) \in K \cdot \lambda \times \mathfrak{p}$ by

$$
\Phi^{\delta}(k \lambda, Z):=k \lambda+\delta \lambda_{0} \circ\left(\sum_{n \geqslant 0} \frac{\operatorname{ad}(Z)^{2 n}}{(2 n) !}\right),
$$

is a moment map for $\left(K \cdot \lambda \times \mathfrak{p}, \Omega^{\delta}\right)$. Consequently, we obtain a moment map for $\Omega_{t}^{\delta}:=t \Gamma^{*} \Omega_{G \cdot \lambda}+(1-t) \Omega^{\delta}$ by taking $\Phi_{t}^{\delta}:=t \Phi_{\Gamma^{*} \Omega_{G \cdot \lambda}}+(1-t) \Phi^{\delta}$.

Theorem 5.2. Let $\delta>0$ and $t \in[0,1]$.

A) The moment map $\Phi_{t}^{\delta}$ satisfies the condition d) of Corollary 3.4.

B) Moreover, if $\delta>b_{\lambda}:=\sup _{\|u\|=1,\|v\|=1}\langle\lambda,[u, v]\rangle$, then the 2 -form $\Omega_{t}^{\delta}:=t \Gamma^{*} \Omega_{G \cdot \lambda}+(1-t) \Omega^{\delta}$ is symplectic.

This result will be proved in sections 5.2 and 5.3 .

Proof of Theorem 5.1. We want to apply Corollary 3.4. Since $\delta>b_{\lambda}$, conditions a) and d) of Corollary 3.4 are implied by assertions B) and A) of Theorem 5.2, respectively. The remaining conditions b) and c) can be easily checked from the formulas of $\Omega_{0}^{\delta}=\Gamma^{*} \Omega_{G \cdot \lambda}$ and $\Omega_{1}^{\delta}=\Omega^{\delta}$ on the submanifold $K \cdot \lambda$. Indeed, we have

$\left.\Omega_{t}^{\delta}\right|_{(k \lambda, 0)}(([k, X], A),([k, Y], B))=\langle\lambda,[X, Y]\rangle+\left\langle t \lambda+(1-t) \delta \lambda_{0},[A, B]\right\rangle$

for all $X, Y \in \mathfrak{k} / \mathfrak{k}_{\lambda}$, all $A, B \in \mathfrak{p}$, and all $t \in[0,1]$. This follows from the fact that $\left.\Psi_{0}\right|_{\mathfrak{p}}=\left.\Psi_{0}^{+}\right|_{\mathfrak{p}}=\left.\mathrm{id}\right|_{\mathfrak{p}}$. q.e.d.

The rest of this section is devoted to the proof of a lemma, which will be used to prove both assertions A) and B) of Theorem 5.2.

We begin by setting some notations. For all $\lambda \in \mathfrak{t}^{*}$, let $H_{\lambda}$ be the unique element of $\mathfrak{t}$ such that

$$
B_{\theta}\left(H_{\lambda}, X\right)=\langle\lambda, X\rangle \quad \forall X \in \mathfrak{g} .
$$

For any noncompact positive root $\beta$, we fix two nonzero vectors $E_{\beta} \in \mathfrak{g}_{\beta}$ and $E_{-\beta} \in \mathfrak{g}_{-\beta}$ such that $E_{-\beta}=\overline{E_{\beta}}$. Then, $E_{\beta}+E_{-\beta}$ and $i\left(E_{\beta}-E_{-\beta}\right)$ are in $\mathfrak{p}$ (that is, they are real vectors). Moreover, the family $\left(E_{\beta}+\right.$ $\left.E_{-\beta}, i\left(E_{\beta}-E_{-\beta}\right)\right)_{\beta \in \mathfrak{R}_{n}^{+}}$is an $\mathbb{R}$-basis of $\mathfrak{p}$, and it is well-known that this 
basis of $\mathfrak{p}$ is orthogonal with respect to the inner product $B_{\theta}$. Moreover, we can choose $E_{\beta}$ and $E_{-\beta}$ such that

$$
B_{\theta}\left(E_{\beta}+E_{-\beta}, E_{\beta}+E_{-\beta}\right)=B_{\theta}\left(i\left(E_{\beta}-E_{-\beta}\right), i\left(E_{\beta}-E_{-\beta}\right)\right)=2
$$

(see $[6,4,1])$.

Lemma 5.3. Let $\lambda, \lambda^{\prime}$ be in $\mathcal{C}_{\text {hol }}$. Then, for all $Z \in \mathfrak{p}$, we have

$$
B_{\theta}\left(H_{\lambda}, \operatorname{ad}(Z)^{2} H_{\lambda^{\prime}}\right) \geq\left(\min _{\beta \in \Re_{n}^{+}} \beta\left(H_{\lambda}\right) \beta\left(H_{\lambda^{\prime}}\right)\right)\|Z\|^{2} .
$$

In particular, if we set $m_{\lambda}=\min _{\beta \in \mathfrak{R}_{n}^{+}} \beta\left(H_{\lambda}\right)$, then

$$
B_{\theta}\left(z_{0}, \operatorname{ad}(Z)^{2} H_{\lambda}\right) \geq m_{\lambda}\|Z\|^{2},
$$

and

$$
B_{\theta}\left(H_{\lambda}, \operatorname{ad}(Z)^{2} H_{\lambda}\right) \geq m_{\lambda}^{2}\|Z\|^{2}
$$

for all $Z \in \mathfrak{p}$.

Proof. Let $Z$ be in $\mathfrak{p}$. Then,

$$
Z=\sum_{\beta \in \Re_{n}^{+}}\left(x_{\beta}^{+}\left(E_{\beta}+E_{-\beta}\right)+x_{\beta}^{-} i\left(E_{\beta}-E_{-\beta}\right)\right),
$$

with $x_{\beta}^{ \pm} \in \mathbb{R}$ for all $\beta \in \mathfrak{R}_{n}^{+}$. Note that (19) implies

$$
B_{\theta}(Z, Z)=\sum_{\beta \in \Re_{n}^{+}} 2\left(\left(x_{\beta}^{-}\right)^{2}+\left(x_{\beta}^{+}\right)^{2}\right) .
$$

Let $H \in \mathfrak{t}$. Since $E_{ \pm \beta}$ is in $\mathfrak{g}_{ \pm \beta}$, we have $\left[H, E_{ \pm \beta}\right]= \pm i \beta(H) E_{ \pm \beta}$. Thus, we deduce the two equalities

$$
\left[H, E_{\beta}+E_{-\beta}\right]=\beta(H)\left(i\left(E_{\beta}-E_{-\beta}\right)\right)
$$

and

$$
\left[H, i\left(E_{\beta}-E_{-\beta}\right)\right]=-\beta(H)\left(E_{\beta}+E_{-\beta}\right) .
$$

Consequently,

$$
[H, Z]=\sum_{\beta \in \Re_{n}^{+}} \beta(H)\left(-x_{\beta}^{-}\left(E_{\beta}+E_{-\beta}\right)+x_{\beta}^{+} i\left(E_{\beta}-E_{-\beta}\right)\right),
$$

for all $H \in \mathfrak{t}$.

Now, let $\lambda, \lambda^{\prime} \in \mathcal{C}_{\text {hol }}$. The elements $H_{\lambda}$ and $H_{\lambda^{\prime}}$ of $\mathfrak{t}$ necessarily verify $\beta\left(H_{\lambda}\right)>0$ and $\beta\left(H_{\lambda^{\prime}}\right)>0$ for all $\beta \in \mathfrak{R}_{n}^{+}$. We have

$$
\begin{aligned}
B_{\theta}\left(H_{\lambda}, \operatorname{ad}(Z)^{2} H_{\lambda^{\prime}}\right) & =B_{\theta}\left(\left[H_{\lambda}, Z\right],\left[H_{\lambda^{\prime}}, Z\right]\right) \\
& =\sum_{\beta \in \Re_{n}^{+}} 2 \beta\left(H_{\lambda}\right) \beta\left(H_{\lambda^{\prime}}\right)\left(\left(x_{\beta}^{-}\right)^{2}+\left(x_{\beta}^{+}\right)^{2}\right) .
\end{aligned}
$$

But $\beta\left(H_{\lambda}\right) \beta\left(H_{\lambda^{\prime}}\right)$ is positive for all $\beta \in \mathfrak{R}_{n}^{+}$; thus one can obtain

$$
B_{\theta}\left(H_{\lambda}, \operatorname{ad}(Z)^{2} H_{\lambda^{\prime}}\right) \geq\left(\min _{\beta \in \Re_{n}^{+}} \beta\left(H_{\lambda}\right) \beta\left(H_{\lambda^{\prime}}\right)\right) B_{\theta}(Z, Z),
$$


by (23). This proves equation (20).

If we take $\lambda^{\prime}=\lambda_{0}$, then $H_{\lambda^{\prime}}=H_{\lambda_{0}}=z_{0}$. Since $\beta\left(z_{0}\right)=1$ for all $\beta \in \mathfrak{R}_{n}^{+}$, we must have $\min _{\beta \in \mathfrak{R}_{n}^{+}} \beta\left(H_{\lambda}\right) \beta\left(z_{0}\right)=m_{\lambda}$, and equation (21) is clear. And finally, equation (22) is induced by the equality $\min _{\beta \in \mathfrak{R}_{n}^{+}}\left(\beta\left(H_{\lambda}\right)^{2}\right)=m_{\lambda}^{2}$, which is true because of the positivity of the numbers $\beta\left(H_{\lambda}\right)$.

q.e.d.

5.2. Proof of claim A) of Theorem 5.2. In this section, we prove that the condition d) of Corollary 3.4 is fulfilled in the setting of Theorem 5.2 for any $\delta>0$ and any $t \in[0,1]$.

We define for any $t \in[0,1]$ the element $\lambda_{t}:=t \lambda+(1-t) \delta \lambda_{0}$ of $\mathcal{C}_{\text {hol }}$, and denote by $H_{\lambda_{t}}=t H_{\lambda}+(1-t) \delta z_{0}$ the associated vector in $\mathfrak{t}$.

Recall that $\Phi_{t}^{\delta}$ is $K$-equivariant, so we only need to consider the points $(\lambda, Z)$ with $Z \in \mathfrak{p}$. Using (17) and (18), we make a first computation:

$$
\begin{aligned}
\left\langle\Phi_{t}^{\delta}(\lambda, Z), H_{\lambda_{t}}\right\rangle= & \left\langle t \Phi_{\Gamma^{*} \Omega_{G \cdot \lambda}}(\lambda, Z)+(1-t) \Phi^{\delta}(\lambda, Z), H_{\lambda_{t}}\right\rangle \\
= & t B_{\theta}\left(H_{\lambda}, \sum_{n \geq 0} \frac{\operatorname{ad}(Z)^{2 n}}{(2 n) !} H_{\lambda_{t}}\right) \\
& +(1-t) \delta B_{\theta}\left(z_{0}, \sum_{n \geq 0} \frac{\operatorname{ad}(Z)^{2 n}}{(2 n) !} H_{\lambda_{t}}\right) \\
& +(1-t)\left\langle\lambda, H_{\lambda_{t}}\right\rangle \\
= & B_{\theta}\left(H_{\lambda_{t}}, \sum_{n \geq 0} \frac{\operatorname{ad}(Z)^{2 n}}{(2 n) !} H_{\lambda_{t}}\right)+(1-t)\left\langle\lambda, H_{\lambda_{t}}\right\rangle .
\end{aligned}
$$

Since $\lambda$ and $\lambda_{t}$ are both in $\mathcal{C}_{\text {hol }}$, the number $\left\langle\lambda, H_{\lambda_{t}}\right\rangle$ is positive, because $\left\langle\lambda, H_{\lambda_{t}}\right\rangle=2 \sum_{\alpha \in \mathfrak{R}^{+}} \alpha\left(H_{\lambda}\right) \alpha\left(H_{\lambda_{t}}\right)>0$. $\operatorname{But} \operatorname{ad}(Z)$ is symmetric for $B_{\theta}$; then

$$
\begin{aligned}
\left\langle\Phi_{t}^{\delta}(\lambda, Z), H_{\lambda_{t}}\right\rangle & \geq \sum_{n \geq 0} \frac{1}{(2 n) !} B_{\theta}\left(\operatorname{ad}(Z)^{n} H_{\lambda_{t}}, \operatorname{ad}(Z)^{n} H_{\lambda_{t}}\right) \\
& \geq \frac{1}{2} B_{\theta}\left(\operatorname{ad}(Z) H_{\lambda_{t}}, \operatorname{ad}(Z) H_{\lambda_{t}}\right) .
\end{aligned}
$$

Equation (22) of Lemma 5.3 yields now

$$
\left\langle\Phi_{t}^{\delta}(\lambda, Z), H_{\lambda_{t}}\right\rangle \geqslant \frac{m_{\lambda_{t}}^{2}}{2}\|Z\|^{2} .
$$

Finally, we get the following inequalities, for all $(k \lambda, Z) \in K \cdot \lambda \times \mathfrak{p}$ :

$$
\left\|\Phi_{t}^{\delta}(k \lambda, Z)\right\| \geq \frac{m_{\lambda_{t}}^{2}}{2\left\|H_{\lambda_{t}}\right\|}\|Z\|^{2} \geq\left(\inf _{t \in[0,1]} \frac{m_{\lambda_{t}}^{2}}{2\left\|H_{\lambda_{t}}\right\|}\right)\|Z\|^{2},
$$

because the norms are $K$-invariant. Note that the constant number $\inf _{t \in[0,1]} \frac{m_{\lambda_{t}}^{2}}{2\left\|H_{\lambda_{t}}\right\|}$ is positive, since $H_{\lambda_{t}}$ is never zero and by continuity 
on the compact set $[0,1]$. Therefore, assertion d) of Corollary 3.4 is checked, and claim A) of Theorem 5.2 is proved.

5.3. Proof of claim B) of Theorem 5.2. It remains to prove that for $\delta$ sufficiently large, the closed 2 -form $\Omega_{t}^{\delta}$ is symplectic. By $K$-invariance of this 2 -form, it is enough to prove that $\Omega_{t}^{\delta}$ is nondegenerate at any point of $\{\lambda\} \times \mathfrak{p}$.

Let $Z \in \mathfrak{p}$. We transform the skew-symmetric bilinear form $\left.\Omega_{t}^{\delta}\right|_{(\lambda, Z)}$ through the invertible linear map $\left.\operatorname{id} \oplus \Psi_{Z}^{+}\right|_{\mathfrak{p}}$ on $\mathfrak{k} / \mathfrak{k}_{\lambda} \oplus \mathfrak{p}$. This requires us to define, for all $Z \in \mathfrak{p}$, the two nondegenerate skew-symmetric bilinear forms $\left.\omega_{0}^{\delta}\right|_{Z}$ and $\left.\omega_{1}\right|_{Z}$ on $\mathfrak{k} / \mathfrak{k}_{\lambda} \oplus \mathfrak{p}$, by

$$
\left.\omega_{0}^{\delta}\right|_{Z}((X, A),(Y, B))=\langle\lambda,[X, Y]\rangle+\delta B_{\theta}\left(z_{0},[A, B]\right),
$$

for any $\delta>0$, and

$$
\left.\omega_{1}\right|_{Z}((X, A),(Y, B))=\left\langle\lambda,\left[X+\chi_{Z}(A), Y+\chi_{Z}(B)\right]\right\rangle+\langle\lambda,[A, B]\rangle,
$$

for all $(X, A),(Y, B) \in \mathfrak{k} / \mathfrak{k}_{\lambda} \oplus \mathfrak{p}$, where the linear map $\chi_{Z}$ is defined by (4).

Now, $\left.\Omega_{t}^{\delta}\right|_{(\lambda, Z)}$ is nondegenerate if and only if $\left.t \omega_{1}\right|_{Z}+\left.(1-t) \omega_{0}^{\delta}\right|_{Z}$ is. Since $\left.\omega_{0}^{\delta}\right|_{Z}$ is clearly nondegenerate, it suffices to prove the next lemma.

Lemma 5.4. Let $Z \in \mathfrak{p}$ and $\delta>0$. If $\delta>b_{\lambda}$, then the skewsymmetric bilinear form $\left.\omega_{1}\right|_{Z}+\left.c \omega_{0}^{\delta}\right|_{Z}$ is nondegenerate for any $c>0$.

Proof. We are going to prove the statement of Lemma 5.4 by contraposition, that is, if the bilinear form $\left.\omega_{1}\right|_{Z}+\left.c \omega_{0}^{\delta}\right|_{Z}$ is degenerate for some $c>0$, then $\delta \leq b_{\lambda}$.

So let $c>0$ be such that $\left.\omega_{1}\right|_{Z}+\left.c \omega_{0}^{\delta}\right|_{Z}$ is degenerate. Then there exists $(X, A) \in \mathfrak{k} / \mathfrak{k}_{\lambda} \oplus \mathfrak{p}$ nonzero such that the equality

$$
\left.\omega_{1}\right|_{Z}((X, A),(Y, B))=-\left.c \omega_{0}^{\delta}\right|_{Z}((X, A),(Y, B))
$$

is valid for all $(Y, B) \in \mathfrak{k} / \mathfrak{k}_{\lambda} \oplus \mathfrak{p}$. First, taking $B=0$, we have

$$
\left\langle\lambda,\left[X+\chi_{Z}(A), Y\right]\right\rangle=-c\langle\lambda,[X, Y]\rangle, \quad \forall Y \in \mathfrak{k} / \mathfrak{k}_{\lambda} .
$$

This yields that $X+\chi_{Z}(A)=-c X \bmod \mathfrak{k}_{\lambda}$, that is, $\chi_{Z}(A)=-(c+1) X$ $\bmod \mathfrak{k}_{\lambda}$. But, by linearity of $\chi_{Z}$, we cannot have $A=0$, because $X$ would also be zero, which would contradict the hypothesis " $(X, A) \neq 0$ ". The equation (24) is now reduced to

$$
\left\langle\lambda,\left[X+\chi_{Z}(A), \chi_{Z}(B)\right]\right\rangle+\langle\lambda,[A, B]\rangle=-c \delta B_{\theta}\left(z_{0},[A, B]\right),
$$

satisfied for all $B \in \mathfrak{p}$. It is equivalent to

$$
\delta B_{\theta}\left(z_{0},[A, B]\right)+\frac{1}{c}\langle\lambda,[A, B]\rangle=\left\langle\lambda,\left[X, \chi_{Z}(B)\right]\right\rangle,
$$


for all $B \in \mathfrak{p}$, since $X+\chi_{Z}(A)=-c X \bmod \mathfrak{k}_{\lambda}$. Taking $B=-\left[z_{0}, A\right] \in \mathfrak{p}$ in $(25)$, we get

$$
\begin{aligned}
0 & <\delta B_{\theta}\left(z_{0},\left[A,-\left[z_{0}, A\right]\right]\right)+\frac{1}{c}\left\langle\lambda,\left[A,-\left[z_{0}, A\right]\right]\right\rangle \\
& \leq-\frac{1}{c+1}\left\langle\lambda,\left[\chi_{Z}(A), \chi_{Z}\left(-\left[z_{0}, A\right]\right)\right]\right\rangle .
\end{aligned}
$$

Note that $\|A\|^{2}=B_{\theta}(A, A)=B_{\theta}\left(z_{0},\left[A,-\left[z_{0}, A\right]\right]\right)$. Moreover, we have $\frac{1}{c}\left\langle\lambda,\left[A,-\left[z_{0}, A\right]\right]\right\rangle=\frac{1}{c} B_{\theta}\left(z_{0}, \operatorname{ad}(A)^{2} H_{\lambda}\right) \geq 0$, from $(21)$ of Lemma 5.3 applied to $A \in \mathfrak{p}$. We deduce the following inequalities:

$$
\begin{aligned}
\delta\|A\|^{2} & \leq \delta B_{\theta}\left(z_{0},\left[A,-\left[z_{0}, A\right]\right]\right)+\frac{1}{c}\left\langle\lambda,\left[A,-\left[z_{0}, A\right]\right]\right\rangle \\
& \leq \frac{1}{c+1}\left\langle\lambda,\left[\chi_{Z}(A), \chi_{Z}\left(\left[z_{0}, A\right]\right)\right]\right\rangle \\
& \leq \frac{1}{c+1} b_{\lambda}\left\|\chi_{Z}(A)\right\| \cdot\left\|\chi_{Z}\left(\left[z_{0}, A\right]\right)\right\| .
\end{aligned}
$$

The number $c$ is positive, so $0<\frac{1}{c+1} \leq 1$. Furthermore, by Lemma 2.2, the linear operator $\chi_{Z}$ is symmetric for $B_{\theta}$ and all its eigenvalues are in ] $-1,1[$. Hence,

$$
\left\|\chi_{Z}(W)\right\| \leq\|W\| \quad \forall W \in \mathfrak{g} .
$$

Consequently, $\left\|\chi_{Z}\left(\left[z_{0}, A\right]\right)\right\| \leq\left\|\left[z_{0}, A\right]\right\|=\|A\|$. So $\delta\|A\|^{2} \leq b_{\lambda}\|A\|^{2}$, with $A \neq 0$. Then we conclude that $\delta \leq b_{\lambda}$. $\quad$ q.e.d.

This concludes the proof of Theorem 5.2.

\section{References}

[1] M. Bordemann, M. Forger \& H. Römer, Homogeneous Kähler manifolds: paving the way towards new supersymmetric sigma models, Comm. Math. Phys. (4) 102 (1986), 605-617, MR 0824094, Zbl 0585.53018.

[2] G. Deltour, Kirwan polyhedron of holomorphic coadjoint orbits, Transform. Groups (2) 17 (2012), 351-392, MR 2921070.

[3] J.J. Duistermaat \& J.A.C. Kolk, Lie groups, Universitext. Springer-Verlag, Berlin, (2000), MR 1738431, Zbl 0955.22001.

[4] S. Helgason, Differential geometry, Lie groups, and symmetric spaces, volume 80 of Pure and Applied Mathematics, Academic Press Inc. [Harcourt Brace Jovanovich Publishers], New York, (1978), MR 0514561, Zbl 0451.53038.

[5] Y. Karshon \& S. Tolman, Centered complexity one Hamiltonian torus actions, Trans. Amer. Math. Soc. (12) 353 (2001), 4831-4861 (electronic), MR 1852084, Zbl 0992.53062.

[6] A.W. Knapp, Lie groups beyond an introduction, volume 140 of Progress in Mathematics, Birkhäuser Boston Inc., Boston, MA, second edition, (2002), MR 1920389, Zbl 1075.22501.

[7] J. Lafontaine, Introduction aux variétés différentielles, EDP Sciences, Les Ulis, (1996), Zbl 0872.53001. 
[8] D. McDuff, The symplectic structure of Kähler manifolds of nonpositive curvature, J. Differential Geom. (3) 28 (1988), 467-475, MR 0965224, Zbl 0632.53058.

[9] J. Moser, On the volume elements on a manifold, Trans. Amer. Math. Soc. 120 (1965), 286-294, MR 0182927, Zbl 0141.19407.

[10] S. Nasrin, Corwin-Greenleaf multiplicity functions for Hermitian symmetric spaces and multiplicity-one theorem in the orbit method, Internat. J. Math. (3) 21 (2010), 279-296, MR 2647001, Zbl 1193.22013.

[11] P.-É. Paradan, Multiplicities of the holomorphic discrete series, preprint (arXiv: 0812.0059).

[12] F.W. Warner, Foundations of differentiable manifolds and Lie groups. Corrected reprint of the 1971 edition, Graduate Texts in Math. 94, Springer-Verlag, New York-Berlin, (1983), MR 0722297, Zbl 0516.58001.

DÉPARTEMENT DE MAThÉmatiques, CC051 Université Montpellier II

Place EugÈne Bataillon

34095 Montpellier Cedex 5, France

E-mail address: guillaume.deltour@math.univ-montp2.fr 